Published in final edited form as:

World J Urol. 2009 February ; 27(1): 75-79. doi:10.1007/s00345-008-0358-9.

\title{
Prognostic factors in T3b renal cell carcinoma
}

\author{
Benjamin J. Coons, Andrew A. Stec, Kelly L. Stratton, Sam S. Chang, Michael S. Cookson, \\ S. Duke Herrell, Joseph A. Smith Jr, and Peter E. Clark \\ Department of Urologic Surgery, Vanderbilt University Medical Center, A-1302 Medical Center \\ North, Nashville, TN 37232-2765, USA \\ Peter E. Clark: peter.clark@vanderbilt.edu
}

\begin{abstract}
Objectives-We reviewed our patients with pathologic T3b renal cell carcinoma (RCC) to determine which factors influenced survival in this high risk patient group.
\end{abstract}

Methods-From April 1988 to August 2006, 722 patients underwent nephrectomy for RCC at Vanderbilt University. 128 patients (17\%) had T3b disease by 2002 AJCC TNM staging criteria. 31 (24\%) of these patients had known metastases at the time of nephrectomy. Patient demographics, clinical, and pathological characteristics were collected.

Results-There were 95 men (74\%) and 33 women (26\%) whose median age was 64 years (range 35-87). Median follow-up was 25.2 months (0-124). Median follow-up among those still alive at last follow up was 45.8 months (2.4-114). For overall survival (OS), disease specific survival (DSS), and recurrence free survival (RFS), non-clear cell histology, grade, presence of sarcomatoid features, LN positive disease, presence of necrosis, positive margins, and metastasis present at the time of nephrectomy were all associated with worse outcomes. Race, gender, ASA class, age, and inferior vena cava (IVC) involvement were not associated with outcome. On multivariate analysis, metastasis at the time of nephrectomy, margin involvement, and the presence of necrosis were independently associated with decreased OS and DSS. The presence of necrosis and lymph node involvement were independent predictors of worse RFS.

Conclusions-Our data suggests that in patients with T3b RCC, the presence or absence of macroscopic necrosis should be included as part of the pathology report to help guide prognosis in this high risk patient group.

\section{Keywords}

Kidney; Renal cell carcinoma; Thrombosis; Prognosis; Necrosis

\section{Introduction}

Renal cell carcinoma (RCC) with renal vein and inferior vena cava (IVC) involvement is discovered in 5-20\% of the 54,000 newly diagnosed RCC's each year [1]. Because of modern operative techniques and post-operative care, most patients with RCC and invasion into the renal vein and IVC are successfully treated through surgical management. Despite excellent survival in the perioperative period, the 5-year survival rate in this patient population without metastasis is only $40-60 \%$ [2-4]. In some studies, 5-year survival rate drops to less than $20 \%$ with the presence of metastatic disease [5].

Correspondence to: Peter E. Clark, peter. clark@vanderbilt .edu.

Conflict of interest statement There is no conflict of interest. 
Predicting prognostic factors in patients with $\mathrm{T} 3 \mathrm{~b} \mathrm{RCC}$ is important for determining future surveillance protocols and treatment strategies as surgical excision of metastatic disease following nephrectomy with thrombectomy gives patients their best chance for survival [6]. In addition, the growing number of targeted agents now available to treat advanced RCC as well as several studies examining the possible role of adjuvant therapy in high risk patients suggests that prognostic factors may play a role in selecting patients for these types of therapy in the future. The TNM staging system is the most widely used classification system for RCC however, there have been many recent suggestions for modification of the staging system based on survival trends in large case series [7,8]. There is a growing appreciation that T3b RCC encompasses a heterogeneous group of tumors that have varying prognoses. A number of different variables including the level of tumor thrombus, vascular wall invasion, sarcomatoid features, and invasion of the perinephric fat have been shown to affect prognosis in some series but not others [7,9]. Due to discrepant and sometimes contradictory findings across studies it has been difficult to come to a consensus as to which variables should be used to further refine prognosis in patients with pT3b RCC. We reviewed our own series of patients with pathologic T3b RCC to determine which factors influence survival in this high risk patient group at our institution.

\section{Methods}

From April 1988 to August 2006, 722 patients underwent nephrectomy for RCC at Vanderbilt University. 128 patients (17\%) had T3b disease by 2002 American Joint Committee on Cancer TNM staging criteria. 31 (24\%) of these patients had known metastases at the time of nephrectomy. We assessed the impact of race, gender, tumor histology, Fuhrman nuclear grade, ASA class, lymph node (LN) status, the presence of metastases, margin status, and the presence of necrosis or sarcomatoid features in the final pathology. Pathology reports that identified necrosis in the gross specimen description were classified as having necrosis present. Tumor thrombus location was classified as involving solely the renal vein or the IVC and renal vein. Due to the relatively small number of patients with non-clear cell histology, analysis of histology was limited to clear cell (conventional) RCC versus non-clear cell histology. Similarly, for grade there were only 3 grade one RCC tumors, so these were grouped with grade two. Thus for Fuhrman grade there were three groups, Grade 1-2 (low grade), Grade 3 (medium) and Grade 4 (high grade). For lymph node status, patients were grouped as either having pathologically proven positive lymph nodes or not. The latter group included patients with radiographically negative lymph node enlargement without evidence of enlarged lymph nodes intra-operatively who did not undergo formal lymphadenectomy and were thus classified as $\mathrm{pNx}$ on final pathology.

The study end points were overall survival (OS), disease specific survival (DSS), and recurrence free survival (RFS). These values were calculated from the date of surgery to either date of last follow up or the relevant event (death, disease specific death, or recurrence). The retrospective nature of this study precluded a standardized follow-up protocol to identify recurrence in our patient population. Nonetheless, most patients at a minimum of every six months underwent chest films, CT scans of the abdomen and pelvis, and comprehensive metabolic panel blood draws for the first three years followed by yearly imaging of the chest, abdomen, and pelvis thereafter. Patients who had not recurred or died were censored at last follow up. For RFS, patients with metastases at the time of nephrectomy were excluded from the analysis. For univariate analyses, the Kaplan-Meier and log-rank method was used to determine factors that impacted OS, DSS, and RFS. Multivariate analyses were accomplished by using a Cox proportional hazard regression model. All analyses utilized commercially available software (Statview, Cary, NC). 


\section{Results}

Radical nephrectomy and tumor thrombectomy was performed in all 128 patients with T3b disease. Pathologic evaluation of the surgical specimens confirmed RCC with either renal vein or renal vein in addition to IVC thrombus. There were 95 men (74\%) and 33 women (26\%) whose median age was 64 (range 35-87) years. Median follow-up was 25.2 months (0-124). Median follow-up among those still alive at last follow up was 45.8 months (2.4-114). Table 1 lists pathologic findings of this patient cohort. Most of the patients were found to have clear cell carcinoma and had tumor venous extension to the level of the IVC in addition to the renal vein. Median tumor size was $9.9 \mathrm{~cm}$ (3.5-21). For the whole group, median OS was 30.8 months, median DSS was 34.3 months, and median RFS was 23.3 months.

On univariate analysis, non-clear cell histology $(P<0.0001)$, grade $(P=0.0063)$, presence of sarcomatoid features $(P=0.0005), \mathrm{LN}$ positive disease $(P<0.0001)$, presence of necrosis $(P$ $<0.0001)$, positive surgical margins $(P=0.0005)$, and metastasis present at the time of nephrectomy $(P<0.0001)$ were all associated with worse OS (see Fig. 1). For DSS, on univariate analysis non-clear cell histology $(P<0.0001)$, grade $(P=0.0007)$, presence of sarcomatoid features $(P=0.0001)$, LN involvement $(P<0.0001)$, necrosis $(P<0.0001)$, positive margins $(P=0.0009)$, and metastasis present at the time of nephrectomy $(P<0.0001)$ were associated with worse outcomes (see Fig. 2). RFS was negatively associated with nonclear cell histology $(P<0.0001)$, grade $(P=0.003)$, LN positive disease $(P<0.0001)$, necrosis $(P<0.0001)$, and sarcomatoid features $(P=0.0001)$ on univariate analysis (see Fig. 3$)$. Race, gender, age, ASA class, and IVC involvement were not found to significantly impact OS, DSS, or RFS on univariate analysis.

On multivariate analysis, metastasis at the time of nephrectomy $(P=0.0002)$, margin involvement $(P=0.0025)$, and the presence of necrosis $(P=0.0005)$ were independently associated with decreased OS (Table 2). These same variables were also independently associated with worsened DSS (Table 3). In addition, the presence of lymph node involvement $(P=0.03)$ and necrosis $(P=0.003)$ were independent predictors of worse RFS (Table 4$)$.

\section{Discussion}

Surgical resection is the mainstay of treatment in patients with RCC involving tumor thrombus. Pathologic staging is a predictor of long-term prognosis in this patient population, however prognosis varies even among tumors within the same stage. We present an analysis of our experience in patients with T3b RCC. We report that the presence of metastasis at the time of nephrectomy was the strongest independent predictor of survival, however other factors such as lymph node positivity and the presence of macroscopic necrosis in the pathological specimen are significant independent prognostic factors when calculating RFS, DSS, and OS.

Many groups have proposed reclassifications of the currently used AJCC TNM staging system based on tumor thrombus extension, however most recent series demonstrate no differences in survival based on the extent of the thrombus [2,5,10-12]. In a prior publication, we have addressed our operative techniques for resection of renal masses with IVC extension [13]. Both in that analysis and in our current series, the level of tumor thrombus did not influence OS, RFS, or DSS.

The presence of necrosis in the pathologic specimen was a poor prognostic indicator in our series both on univariate and multivariate analyses. This finding of necrosis is infrequently addressed in renal cell carcinoma case series. A notable exception is a review addressing the presence of necrosis in a large series similar to ours published from the group at the Mayo Clinic. Their retrospective review showed a significant impact on cancer specific survival when necrosis was present in the pathologic specimen [5]. In their series, the 5-year cancer specific 
survival rates for patients with and without tumor necrosis were 26 and $61 \%$, respectively $(P$ $<0.001)$. Necrosis is a frequent finding in high stage renal cell carcinoma yet because it is infrequently analyzed, most centers do not consider this a prognostic factor. We believe that necrosis should be included as part of the pathology report in order to help guide prognosis in this high risk patient group.

These results need to be confirmed in large, randomized, prospective trials, however we believe that the presence of necrosis in a pathologic specimen should prompt closer follow-up in this high risk patient group. The potential advantageous effects of different adjuvant treatments such as tyrosine kinase inhibitors are becoming more apparent with time. A phase III randomized prospective trial comparing sorafenib, sunitinib, and placebo is now ongoing that may help address the need for adjuvant treatment in high risk patient populations such as ours. However, until further data is available, we do not advocate using systemic, adjuvant treatment in T3b patients with necrosis on pathologic review.

Our study is limited by both its retrospective nature and single institution experience and therefore carries with it all the inherent potential issues associated with all such studies. In addition, although all pathology was done at our institution, there were multiple different pathologists who read and interpreted the specimens. While this carries a risk of introducing bias into the results that are hard to correct for, it also is more representative of a general urology practice. Because this analysis was performed retrospectively, factors such as the percentage of necrosis involving the tumor and comments on microscopic necrosis could not be reliably evaluated.

Despite these limitations, we feel that our results are important in demonstrating the prognostic impact of multiple factors in a large cohort of modern patients with T3b renal cell carcinoma over a period of 18 years. Although more study is needed to validate these results, we believe that the presence of necrosis in a RCC specimen should be noted in the pathology report as it can aid in predicting prognosis after nephrectomy and allow one to better tailor surveillance protocols and post-operative treatment.

\section{Acknowledgments}

The project described was supported by Award Number K08 CA113452 (P.E.C) from the National Institutes of Health. The content is solely the responsibility of the authors and does not necessarily represent the official views of the National Institutes of Health.

\section{References}

1. Jemal A, Siegel R, Ward E, Yongping H, Xu J, Murray T, Thun M. Cancer statistics, 2008. CA Cancer J Clin 2008;58:71-96.10.3322/CA.2007.0010 [PubMed: 18287387]

2. Blute ML, Leibovich BC, Lohse CM, Cheville JC, Zincke H. The Mayo Clinic experience with surgical management, complications and outcome for patients with renal cell carcinoma and venous tumour thrombus. BJU Int 2004;94(1):33-41.10.1111/j.1464-410X.2004.04897.x [PubMed: 15217427]

3. Tanaka M, Fujimoto K, Okajima E, Tanaka N, Yoshida K, Hirao Y. Prognostic factors of renal cell carcinoma with extension into inferior vena cava. Int J Urol 2008;15(5):394-398.10.1111/j. 1442-2042.2008.02017.x [PubMed: 18452454]

4. Trombetta C, Liguori G, Bucci S, Benvenuto S, Garaffa G, Belgrano E. Evaluation of tumor thrombi in the inferior vena cava with intraoperative ultrasound. World J Urol 2007;25(4):381-384.10.1007/ s00345-007-0191-6 [PubMed: 17609962]

5. Zisman A, Wieder JA, Pantuck AJ, et al. Renal cell carcinoma with tumor thrombus extension: biology, role of nephrectomy and response to immunotherapy. J Urol 2003;169(3):909-916.10.1097/01.ju. 0000045706.35470.1e [PubMed: 12576811] 
6. Kavolius JP, Mastorakos DP, Pavlovich C, Russo P, Burt ME, Brady MS. Resection of metastatic renal cell carcinoma. J Clin Oncol 1998;16(6):2261-2266. [PubMed: 9626229]

7. Klatte T, Pantuck AJ, Riggs SB, et al. Prognostic factors for renal cell carcinoma with tumor thrombus extension. J Urol 2007;178(4 pt 1):1189-1195. (discussion 95). 10.1016/j.juro.2007.05.134 [PubMed: 17698087]

8. Leibovich BC, Cheville JC, Lohse CM, et al. Cancer specific survival for patients with pT3 renal cell carcinomacan the 2002 primary tumor classification be improved? J Urol 2005;173(3):716719.10.1097/01.ju.0000151830.27750.d2 [PubMed: 15711250]

9. Ficarra V, Artibani W. Staging system of renal cell carcinoma: current issues. Eur Urol 2006;49(2): 223-225.10.1016/j.eur-uro.2005.11.018 [PubMed: 16426739]

10. Libertino JA, Zinman L, Watkins E Jr. Long-term results of resection of renal cell cancer with extension into inferior vena cava. J Urol 1987;137(1):21-24. [PubMed: 3795361]

11. Hatcher PA, Anderson EE, Paulson DF, Carson CC, Robertson JE. Surgical management and prognosis of renal cell carcinoma invading the vena cava. J Urol 1991;145(1):20-23. discussion 34. [PubMed: 1984092]

12. Sosa RE, Muecke EC, Vaughan ED Jr, McCarron JP Jr. Renal cell carcinoma extending into the inferior vena cava: the prognostic significance of the level of vena caval involvement. J Urol 1984;132 (6):1097-1100. [PubMed: 6502795]

13. Parekh DJ, Cookson MS, Chapman W, et al. Renal cell carcinoma with renal vein and inferior vena caval involvement: clinicopathological features, surgical techniques and outcomes. J Urol 2005;173 (6):1897-1902.10.1097/01.ju.0000158459.42658.95 [PubMed: 15879771] 

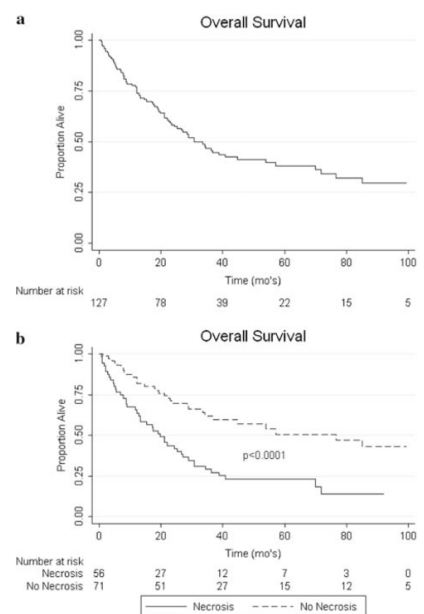

Fig. 1.

a Kaplan-Meier curve for OS. b Impact of necrosis on OS 

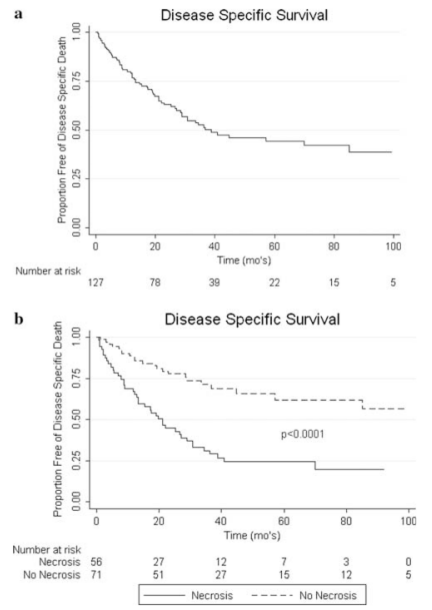

Fig. 2.

a Kaplan-Meier curve for DSS. b Impact of necrosis on DSS 


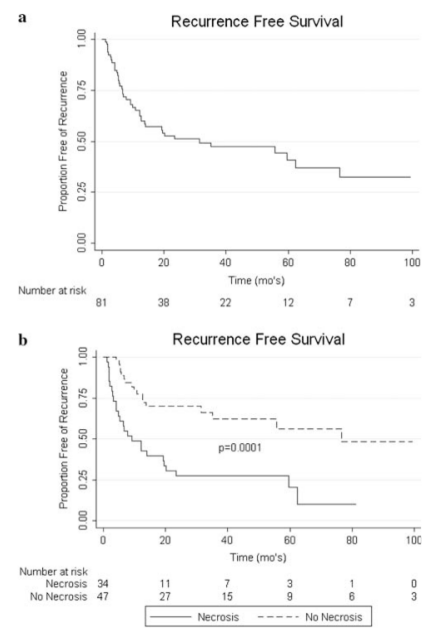

Fig. 3.

a Kaplan-Meier curve for RFS. b Impact of necrosis on RFS 


\section{Table 1}

T3b renal cell pathologic findings

\begin{tabular}{ll}
\hline Histology & \\
Clear cell & $105(82 \%)$ \\
Non-clear cell & $23(18 \%)$ \\
\multicolumn{2}{c}{ Sarcomatoid elements } \\
Yes & $18(14 \%)$ \\
No & $110(86 \%)$ \\
Necrosis present & \\
Yes & $57(44 \%)$ \\
No & $71(66 \%)$ \\
Fuhrman grade & \\
I & $3(2 \%)$ \\
II & $37(29 \%)$ \\
III & $51(40 \%)$ \\
IV & $32(25 \%)$ \\
Unk & $5(4 \%)$ \\
Lymph node & \\
Pos & $21(16 \%)$ \\
Neg & $107(84 \%)$ \\
IVC involvement & \\
Yes & $78(61 \%)$ \\
No & $50(39 \%)$ \\
\hline
\end{tabular}

World J Urol. Author manuscript; available in PMC 2010 October 25. 
Table 2

Overall survival-multivariate analysis

\begin{tabular}{llll}
\hline & HR exp (coef) & $\mathbf{9 5 \%}$ CI & $\boldsymbol{P}$ value \\
\hline Convention (clear cell) & 0.553 & $0.220-1.395$ & 0.2097 \\
Grade & & & \\
$\quad$ Medium (grade 3) & Referent & - & - \\
$\quad$ High (grade 4) & 0.832 & $0.337-2.057$ & 0.6909 \\
$\quad$ Low (grade 1-2) & 0.643 & $0.315-1.315$ & 0.2267 \\
Non-sarcomatoid & 0.989 & $0.369-2.650$ & 0.9828 \\
No LN metastases & 0.535 & $0.236-1.213$ & 0.1344 \\
No Necrosis & 0.333 & $0.152-0.560$ & 0.0002 \\
No Mets at Nx & 0.291 & $0.190-0.582$ & 0.0001 \\
Negative Margins & 0.304 & $0.139-0.666$ & 0.0029 \\
\hline
\end{tabular}


Table 3

Disease specific survival-multivariate analysis

\begin{tabular}{llll}
\hline & HR exp (coef) & $\mathbf{9 5 \%}$ CI & $\boldsymbol{P}$ value \\
\hline Convention (clear cell) & 0.553 & $0.220-1.395$ & 0.2097 \\
Grade & & & \\
$\quad$ Medium (grade 3) & Referent & - & - \\
$\quad$ High (grade 4) & 0.832 & $0.337-2.057$ & 0.6909 \\
$\quad$ Low (grade 1-2) & 0.643 & $0.315-1.315$ & 0.2267 \\
Non-sarcomatoid & 0.989 & $0.369-2.650$ & 0.9828 \\
No LN metastases & 0.535 & $0.236-1.213$ & 0.1344 \\
No Necrosis & 0.333 & $0.152-0.560$ & 0.0002 \\
No Mets at Nx & 0.291 & $0.190-0.582$ & 0.0001 \\
Negative Margins & 0.304 & $0.139-0.666$ & 0.0029 \\
\hline
\end{tabular}

World J Urol. Author manuscript; available in PMC 2010 October 25. 
Table 4

Recurrence free survivial-multivariate analysis

\begin{tabular}{llll}
\hline & HR exp (Coef) & $\mathbf{9 5 \%}$ CI & $\boldsymbol{P}$ value \\
\hline Conventional (Clear cell) & 0.323 & $0.073-1.424$ & 0.1355 \\
Grade & & & \\
$\quad$ Medium (grade 3) & Referent & - & - \\
$\quad$ High (grade 4) & 1.105 & $0.385-3.171$ & 0.8523 \\
$\quad$ Low (grade 1-2) & 0.688 & $0.282-1.680$ & 0.4122 \\
Non-sarcomatoid & 0.767 & $0.184-3.192$ & 0.7152 \\
No LN metastases & 0.257 & $0.075-0.882$ & 0.0307 \\
No Necrosis & 0.295 & $0.130-0.674$ & 0.0037 \\
\hline
\end{tabular}

